\title{
The Impact of Error-Management Climate, Error Type and Error Originator on Auditors' Reporting Errors Discovered on Audit Work Papers
}

\author{
Anna Gold, Ulfert Gronewold, and Steve Salterio
}

\begin{tabular}{|l|l|}
\hline \multicolumn{2}{|l|}{ ERIM REPORT SERIES RESEARCH IN MANAGEMENT } \\
\hline ERIM Report Series reference number & ERS-2010-035-FA \\
\hline Publication & August 2010 \\
\hline Number of pages & 44 \\
\hline Persistent paper URL & \\
\hline Email address corresponding author & agold@rsm.nl \\
\hline Address & Erasmus Research Institute of Management (ERIM) \\
& RSM Erasmus University / Erasmus School of Economics \\
& Erasmus Universiteit Rotterdam \\
& P.O.Box 1738 \\
& 3000 DR Rotterdam, The Netherlands \\
& Phone: + 31 10 408 1182 \\
& Fax: + 31104089640 \\
& Email: info@erim.eur.nl \\
& Internet: www.erim.eur.nl \\
\hline
\end{tabular}

Bibliographic data and classifications of all the ERIM reports are also available on the ERIM website: www.erim.eur.nl 


\section{ERASMUS RESEARCH INSTITUTE OF MANAGEMENT}

\section{REPORT SERIES}

\section{RESEARCH IN MANAGEMENT}

\begin{tabular}{|c|c|}
\hline \multicolumn{2}{|c|}{ ABSTRACT AND KEYWORDS } \\
\hline Abstract & $\begin{array}{l}\text { We examine factors affecting the auditor's willingness to report their own or their peers' self- } \\
\text { discovered errors in working papers subsequent to detailed working paper review. Prior research } \\
\text { has shown that errors in working papers are detected in the review process; however, such } \\
\text { detection rates only rarely exceed } 50 \% \text { of the seeded errors. Hence, measures that encourage } \\
\text { auditors to be alert to their own (or their peers') potential errors any time they revisit the audit } \\
\text { working papers may be valuable in detecting such residual errors and potentially correcting them } \\
\text { before damage occurs to the audit firm or its client. We hypothesize that three factors affect the } \\
\text { auditor's willingness to report post detailed review discovered errors: the local office error } \\
\text { management climate (open versus blame), the type of error (mechanical versus conceptual) and } \\
\text { who committed the error (the individual who committed the error (self) or a peer). Local office } \\
\text { error-management climate is said to be open and supportive where errors and mistakes are } \\
\text { accepted as part of everyday life as long as they are learned from and not repeated. In } \\
\text { alternative, a blame error-management climate focuses on a "get it right the first time" culture } \\
\text { where mistakes are not tolerated and blame gets attached to those admitting to or found } \\
\text { committing such errors. We find that error-management climate has a significant overall effect on } \\
\text { auditor willingness to report errors, as does who committed the error originally. We find both } \\
\text { predicted and unpredicted significant interactions among the three factors that qualify these } \\
\text { observed significant main effects. We discuss implications for audit practice and further } \\
\text { research. }\end{array}$ \\
\hline Free Keywords & audit quality, adjustments, materiality, review \\
\hline Availability & $\begin{array}{l}\text { The ERIM Report Series is distributed through the following platforms: } \\
\text { Academic Repository at Erasmus University (DEAR), DEAR ERIM Series Portal } \\
\text { Social Science Research Network (SSRN), SSRN ERIM Series Webpage } \\
\text { Research Papers in Economics (REPEC), REPEC ERIM Series Webpage }\end{array}$ \\
\hline Classifications & $\begin{array}{l}\text { The electronic versions of the papers in the ERIM report Series contain bibliographic metadata } \\
\text { by the following classification systems: } \\
\text { Library of Congress Classification, (LCC) } \underline{\text { LCC Webpage }} \\
\text { Journal of Economic Literature, (JEL), JEL Webpage } \\
\text { ACM Computing Classification System CCS Webpage } \\
\text { Inspec Classification scheme (ICS), ICS Webpage }\end{array}$ \\
\hline
\end{tabular}


The Impact of Error-Management Climate, Error Type and Error Originator on Auditors' Reporting Errors Discovered on Audit Work Papers*

\author{
Anna Gold \\ Assistant Professor \\ Rotterdam School of Management \\ Erasmus University (The Netherlands) \\ Ulfert Gronewold \\ PricewaterhouseCoopers Junior Assistant Professor \\ Ruhr University Bochum (Germany) \\ Steve Salterio \\ Professor and PricewaterhouseCoopers/Thomas O'Neill Faculty Fellow in Accounting \\ School Of Business \\ Queen's University (Canada)
}

Preliminary: Your comments are welcome.

\begin{abstract}
August 10, 2010
*We thank the partners and staff at the six German based audit firms for assisting us with this research. We thank Myra Landsburg, Andy Grien, Joan Luft and Michel Seadle for proofing and commenting on the English to German translation and to Sarah Reuter for professionally retranslating the material. We thank the participants at the University of Texas El Paso accounting workshop and Jim Hunton for comments on this preliminary draft.
\end{abstract}




\title{
The Impact of Error-Management Climate, Error Type and Error Originator on Auditors' Reporting Errors Discovered on Audit Work Papers
}

\begin{abstract}
We examine factors affecting the auditor's willingness to report their own or their peers' selfdiscovered errors in working papers subsequent to detailed working paper review. Prior research has shown that errors in working papers are detected in the review process; however, such detection rates only rarely exceed $50 \%$ of the seeded errors. Hence, measures that encourage auditors to be alert to their own (or their peers') potential errors any time they revisit the audit working papers may be valuable in detecting such residual errors and potentially correcting them before damage occurs to the audit firm or its client. We hypothesize that three factors affect the auditor's willingness to report post detailed review discovered errors: the local office errormanagement climate (open versus blame), the type of error (mechanical versus conceptual) and who committed the error (the individual who committed the error (self) or a peer). Local office error-management climate is said to be open and supportive where errors and mistakes are accepted as part of everyday life as long as they are learned from and not repeated. In alternative, a blame error-management climate focuses on a "get it right the first time" culture where mistakes are not tolerated and blame gets attached to those admitting to or found committing such errors. We find that error-management climate has a significant overall effect on auditor willingness to report errors, as does who committed the error originally. We find both predicted and unpredicted significant interactions among the three factors that qualify these observed significant main effects. We discuss implications for audit practice and further research.
\end{abstract}




\section{The Impact of Error-Management Climate, Error Type and Error Originator on Auditors' Reporting Errors Discovered on Audit Work Papers}

\section{INTRODUCTION}

Auditors, while highly competent professionals who tend to be less biased in areas of their professional expertise than people are in general (Smith and Kida 1991), are fallible human beings who make, and sometimes later discover, mistakes they made. The within audit firm review process is designed to detect errors as part of the firm's quality control process; however, auditors may discover errors made by themselves or their peers in the working papers, even after the review process is completed. ${ }^{1}$ To date, no research has examined how auditors deal with the discovery of own or peers' errors post review. Hence, given the existence of such error discovery, the goal of our research is to examine the factors that encourage or discourage auditors to report the discovery of these post-review discovered errors within the audit firm. We identify three factors that may affect the willingness to report discovered errors: the organization's error-management climate, the type of audit error and whether the error is made by the auditor or by a peer.

Psychology research (e.g., Van Dyck et al. 2005) suggests that the organization's errormanagement climate affects how auditors deal with self-discovered errors. At one extreme, the climate can be one of openness toward the possibility that errors will be made and support provided for learning from the mistakes when errors are reported, committed by either oneself or others. In a blame climate, discovery and reporting of a mistake made would lead to sanctions on the person who committed the error. Research in organizations more broadly suggests that organizations that are open and accepting of error detection have less difficulty in encouraging

\footnotetext{
${ }^{1}$ For evidence of existence of such working paper errors after within firm review consult any Public Company Accounting Oversight Board (PCAOB) report on public company audits (e.g. PCAOB 2004).
} 
such reports of error discovery, whereas organizations where a "get it right the first time" is the dominant culture have a lower likelihood of such reporting (e.g., Van Dyck et al. 2005).

A second factor that may affect how these post-review self-discovered errors are dealt with is error type. Prior audit research (e.g., Ramsay 1994) has identified two major classes of errors in working papers: mechanical and conceptual. Mechanical errors are the result of incorrect computations or transfers of numbers that are readily available in the audit file whereas conceptual errors deal with misapplication of audit procedures or misinterpretation of results of such procedures, such that further work is not done while it is called for from the test results. We suggest that error type moderates the effect error-management climate has on an auditor's willingness to report an error.

A third factor we consider is whether the discovered error was committed by the auditor or by a peer ('error originator'). Research on whistle-blowing (i.e., the tendency to report unlawful actions such as fraud, pollution etc.) shows that most people witnessing such actions do not report them (e.g., Miceli et al. 2009). Hence, even in these serious settings, the whistleblowing research suggests that the willingness to report on a peer would be lower than willingness to report on oneself, because of the implicit social norms against being a "tattletale" or a "snitch" (Pershing 2003). Hence, we believe that an auditor would be more willing to report his/her own error than that of a peer.

We examine these three factors and interactions among them via an experiment with over 190 auditors who discover an error in the working papers that is highly suggestive of a material misstatement in the client's financial statements. We focus on errors detected by auditors that are suggestive of a material misstatement in the client's financial statements (as opposed to misapplying an audit technique that has no direct consequences for financial statement quality) 
and discovered after all detailed file review has been completed but before the financial statements have been released (see SQCS10 (AICPA 2009) and ISQC1 (IAASB 2009)). Hence, the errors are potentially correctible via the audit firm carrying out additional audit work to ensure the error requires adjustment and the client firm correcting the financial statements before they are released, or the audit firm issues a modified audit opinion if the client firm declines additional auditing or correction of the financial statements.

The experimental results demonstrate that office error-management climate and error originator affect an auditor's willingness to report the post-review discovered error. In particular, an open climate encourages more willingness to report than a blame climate and self-committed errors are more likely to be reported than those of peers. However, these findings are qualified by interactions of the two factors with error type in that both effects are found for mechanical errors discovered but not for conceptual errors. Further, the two factors, climate and originator, interact in that the originator effect holds for a blame error management condition but not for the open climate condition, whereas the climate effect holds for a peer's error discovered but not own errors discovered.

These findings suggest that audit firms need to consider the degree of flexibility that they give local office management in setting the error management climate for the office. In particular, thought needs to be given to offices that are managed with a culture of "getting it right the first time" (or blame) as their very emphasis on perfection may well lead to errors known to junior auditors not being reported. Further, our research draws attention to the observation that absent a clear role as a reviewer, an auditor discovering a working paper error is more likely to report his own errors as opposed to other auditor's errors. Having an open error-management 
climate mitigates this latter effect, reemphasizing the need to consider office management practices effects on audit quality.

Prior audit research (e.g., Ramsay 1994) focuses on the review process to detect errors in working paper files; however, this literature does not address self-discovery of errors as it has focused on the hierarchical nature of working paper review (e.g., Asare and McDaniel 1996; Bamber and Ramsay 1997; Tan and Trotman 2003). Further this literature documents, even in highly simplified experimental settings, that the review process rarely discovers more than 50 percent of the errors, thus leaving substantial room for self detection and reporting of errors subsequent to review to improve audit quality. Further, prior audit research (e.g., McDaniel 1990) on audit firm organizational issues (e.g., structure, time pressure etc.) tends to focus on what causes auditors to commit quality reducing procedures (e.g., Alderman and Deitrick 1982 examine signing off on audit programs of procedures not done) or the reduction in the ability of the auditor to effectively carrying out the audit procedures due to an organizational factor (e.g., McDaniel 1990 focuses on time pressure). However, that literature has not examined organizational error-management climate for its overall effects on audit quality. Hence, it is important to study auditors' willingness to report self-detected errors because encouraging auditors to report these errors may result in fewer mistakes in working papers and greater quality in the client's financial reporting, especially in cases where the errors are discovered post detailed working paper review.

The remainder of this paper is organized as follows: the next section provides background information about the review process and self-monitoring leading to the development of five hypotheses. We then present the research design for an experiment to test these hypotheses. Results including manipulation checks and robustness checks are reported. The paper concludes 
with a discussion of results and their implications for audit practice and future research in the final section.

\section{BACKGROUND AND HYPOTHESES DEVELOPMENT}

\section{The Review Process and Self-Monitoring}

The purpose of the working paper review process is to detect errors made by subordinate auditors. Reviewers' error detection rates depend on the reviewer's rank and the type of error, such that senior reviewers are better than manager reviewers in detecting mechanical errors (i.e., relatively objective, verifiable, and concrete errors, requiring little or no judgment to detect them), whereas the opposite applies for conceptual errors (i.e., relatively subjective, unverifiable, and imprecise errors, requiring substantial judgment to detect them) (e.g., Ramsay 1994; Owhoso et al. 2002). Harding and Trotman (1999) found this pattern to generalize to lower ranks as well, showing that staff auditors were even better than seniors in detecting mechanical errors, but less effective in detecting conceptual errors. Further, reviewers' industry specialization appears to be a requirement for effective error detection (Owhoso et al. 2002 report a 75\% detection rate for industry specialist reviewers detecting common industry working paper errors), and allencompassing reviews lead to higher detection rates than specialized reviews that focus only on a specific type of error (Bamber and Ramsay 1997; 2000). Detection rates are also affected by task complexity, the reviewer's familiarity with the preparer (Asare and McDaniel 1996), the preparer's work paper stylization (differential emphasis on different types of errors), and the reviewer's sensitivity to those stylization attempts (Tan and Trotman 2003).

Significant audit research has shown the review process to be a strong accountabilityinducing mechanism, such that better quality audit work is carried out in its presence as compared to its absence (e.g., Kennedy 1993; Gibbins and Newton 1994; Brazel et al. 2004). 
However, we also note that overall error detection rates of the working paper review process rarely exceed 50 percent even in highly simplified experimental settings (e.g., Asare and McDaniel 1996; Bamber and Ramsay 1997; Tan and Trotman 2003), while in somewhat more realistic settings, error detection rates even drop to under 50 percent (Owhoso et al. 2002). This evidence suggests that enhancing self-review and encouraging auditors to report the results of that self-review may result in a greater ability of audit firms to detect errors in their working papers prior to issuing the audit report and hence increase the quality of their client's financial reporting.

Prior research shows that auditors, even at the junior staff level, have the ability to detect at least some of their own errors (e.g., Harding and Trotman 1999), so why might they not report them? Prior research suggests that there are costs to an auditor who discovers and fixes an error prior to the commencement of the review process. For example, he may exceed the time budget or have negative performance evaluations if a more senior auditor is aware of the behavior (e.g., Alderman and Deitrick 1982; Kelley and Margheim 1990; Malone and Roberts 1996; McNair 1991; Pierce and Sweeney 2006; Willett and Page 1996). After the audit review process commences the "costs" of reporting these self-discovered errors would be higher due to their greater visibility affecting auditors' own performance evaluations and, in addition, reporting these errors would expose their supervisors who reviewed the files to criticism (Rothschild and Miethe 1999). Indeed, some research suggests that audit seniors are rarely concerned about the (un)ethicality of failing to report errors they made in working papers (Pierce and Sweeney 2006).

A related question is given that the auditor is not instructed to do further review of their own working papers, how could such post-review errors come to be discovered? Auditors are frequently asked to consult the audit files on related questions, to tidy up audit files for storage 
purposes or for second partner review among many other reasons that a junior auditor might be re-reading reviewed working papers. A rich body of psychological and neurological research shows that people naturally perform self-monitoring in order to detect and correct possible errors in everyday life, thus not requiring that the auditor explicitly be told to check for errors at these times but rather such self-monitoring for errors occurs naturally. Research shows that a basic level of self-monitoring occurs automatically and consistently to control one's own behavior (e.g., Ganushchak and Schiller 2006; 2008a; 2008b; Rabbitt 1990; Schneider and Shiffrin 1977; Shiffrin and Schneider 1977; Vanleuvan and Wang 1997). ${ }^{2}$ Such monitoring occurs even in situations where people are explicitly instructed to ignore any possible errors that they may have committed (Rabbitt 1969; 1990), demonstrating that error self-monitoring is an automatic human response that cannot be readily suppressed.

Translating these findings from psychology to the audit environment suggests that auditors should naturally carry out self-monitoring of their work, regardless of the stage they are at in the audit process and no matter why they are engaged in working with the audit file. Due to the unique in-depth knowledge of their own work, we believe that auditors can discover their own errors that may go undetected by others including the work paper reviewers. ${ }^{3}$

\section{Error-Management Climate}

Psychology research suggests that an organization's error-management climate is an important determinant of organizational members' willingness to report errors that they have committed and detected (Van Dyck et al. 2005). Organizational error-management climate comprises shared beliefs, norms, and common practices regarding the management of errors in the organization

\footnotetext{
${ }^{2}$ This mechanism has been studied in various areas, including verbal monitoring (e.g., Ganushchak and Schiller 2006, 2008a and 2008b; Hartsuiker and Kolk 2001; Levelt 1983 and 1989; Nooteboom and Quené 2008; Postma 2000) and action and performance monitoring (e.g., Desmurget and Grafton 2000; Luu et al. 2000; RodríguezFornells et al. 2002).

${ }^{3}$ This was also confirmed during numerous discussions with audit practitioners.
} 
(Van Dyck et al. 2005). Research has shown that organizations differ in their error-management climates, which can be characterized by different degrees of "error management" (Van Dyck 1997 and 2009).

A high error-management climate (henceforth called "open climate") implies that errors are recognized as likely to occur whenever fallible humans carry out tasks and hence openly supports ways to handle the discovery of such errors. Indications that such an open climate exists in an organization include open discussion of errors with others, obtaining others' help after discovering errors, thorough analysis of errors and their potential causes, and concern with getting errors corrected. This open climate has been shown in non-audit settings to help develop a thorough understanding about errors, their causal antecedents and their effective handling among the organization's members (via reporting and communicating about errors), so that such a climate can be expected to be beneficial to the overall quality and performance of the organization (Van Dyck et al. 2005) versus an organization that does not have such a climate. ${ }^{4}$ Additional characteristics such research has identified as indicating an open climate include upper management being positive toward the communication about errors and preventing individuals from being punished for reporting such errors (Edmondson 1999; Van Dyck et al. 2005; Van Dyck 2009).

In the opposite climate, which we call a blame climate (known in the literature as "low error management" or "error aversion"), the above-mentioned practices are only paid "lip service" in the organization (Van Dyck 1997 and 2009; Van Dyck et al. 2005). At the extreme, in blame organizations, any reported error may lead people to believe that there will be sanctions,

\footnotetext{
${ }^{4}$ For the positive effect of communicating about errors on effective organizational learning also Homsma et al. 2009.
} 
no matter how small the error reported (e.g., Rybowiak et al. 1999; Van Dyck 2009; Van Dyck et al. 2005).

We suggest that audit firms' offices may also vary with regard to their error-management climate. Error-management climate refers to a firm's employees' perception of actual practices and local interpretation of official firm wide policies. Hence, in an audit firm, error-management climate will likely depend on the local office culture; potentially following the managing partner's or head of audit practice's leadership style (see Covaleski et al. 1998; Dirsmith and Covaleski, 1985). In some large offices there could even be audit team effects (Covaleski et al. 1997). Prior audit research has shown that organizational differences affect auditors' behavior (e.g., Bowrin 1998; Hermanson 1997; Hyatt and Prawitt 2001) and recent archival audit research has shown differences in audit quality between audit firm offices (e.g. Ferguson et al. 2003; Francis et al. 1999; Reynolds and Francis 2000), reinforcing our argument. Gronewold and Donle (2010) have shown that auditors perceive differences in error-management climate in diverse audit organizations (e.g., Big-4 and smaller audit firms, internal audit departments, government auditing, etc.).

Based on the previous discussion, an open climate is more likely to encourage auditors to report errors that they have detected whereas a blame climate is more likely to result in a lower willingness of auditors to report such errors. We therefore expect the following main effect for organizational error-management climate, ceteris paribus:

H1: Auditors are more willing to report post-review discovered errors in an open errormanagement climate than in a blame climate.

\section{Interaction between Error-Management Climate and Error Type}

Consistent with prior audit research (e.g., Ramsay 1994), we differentiate between two error types: mechanical and conceptual. Mechanical errors are defined as relatively objective, 
verifiable, and concrete errors, requiring little or no judgment to detect them (e.g., Ramsay 1994;

Owhoso et al. 2002). Mechanical errors are relatively easy to detect because salient cues are typically present, which facilitate their discovery. An example of a mechanical error would be an erroneous calculation (e.g., an analytical procedure with an arithmetic error in it); here, the presence of the procedure and its calculation is a relatively salient cue for discovering the error via re-performance of the calculation. In contrast, conceptual errors are relatively subjective, unverifiable, imprecise, and require substantial judgment to be detected (e.g., Ramsay 1994; Owhoso et al. 2002). Cues that would facilitate the detection of conceptual errors are often absent or at least less salient. For example, a conceptual error would be a required calculation (e.g., an analytical procedure), which was not carried out at all. In this case, finding such an error rather requires a significant degree of judgment and relevant task knowledge to know that a procedure should be carried out in the circumstances. ${ }^{5}$

We predict that the effect of error-management climate on reporting willingness will be greater for mechanical errors than for conceptual errors, for reasons of varying future competence attributions among staff auditors (Covaleski et al. 1997). Considering mechanical errors, the reviewer may perceive such errors as a sign of somewhat "careless" work, which can easily be rationalized away because such errors can potentially happen even to a competent auditor. As such, the mechanical error is unlikely to be attributed to a lack of underlying competence, but rather, it can be attributed to engagement-related pressures prevailing during most audits (e.g., time pressure (McDaniel 1990)). Consequently, an auditor would probably

\footnotetext{
${ }^{5}$ Most audit research makes use of the distinctions between mechanical and conceptual errors based on the original categorization of error types by Ramsay (1994). Alternative characterizations of error types can be readily reconciled to this initial classification. For example, Tan and Trotman (2003) distinguish between documentation errors (which largely correspond to mechanical errors) and conclusion errors (which correspond to conceptual errors), while Asare and McDaniel (1996) differentiate classification (related to mechanical errors) from conclusion errors (conceptual errors).
} 
expect a relatively mild rebuke by the superior and would therefore be highly receptive to the influence of the prevailing error-management climate. Hence, in an open climate, auditors should be highly willing to report the mechanical error, because there are no or very few repercussions to be concerned about. Not only does the climate promote open dealing with errors, but also is the nature of the error less likely to be attributed to professional incompetence.

On the other hand, given the detection of a conceptual error, the auditor may be concerned that the superior considers the error as far more serious, since it could be attributed to an overall lack of staff auditor competence. Engagement-related pressures cannot as easily excuse an auditor's misinterpreting or misapplying of fundamental audit knowledge. In this case, the auditor would be more worried about the repercussions resulting from the reporting of the error to the superior, since the superior might attribute the error to the auditor's lacking competence, which in turn might seriously damage the auditor's reputation. Consequently, we argue that this auditor concern will largely dominate the potentially favorable effect on reporting willingness that an open error-management climate might have (see H1), because auditors will be concerned about their professional reputation even when the climate is such that errors are accepted. Hence, even in an open climate, auditors may fear repercussions of reporting a conceptual error, since superiors may attribute the error to the auditor's professional incompetence.

In a blame climate, however, we expect auditors to be significantly less willing to report errors of either type as compared to an open climate. This is due to the attribution of all mistakes to personal incompetence on the part of the person who commits the error (i.e., a culture of "getting it right the first time" does not allow for failure). Hence, because of the climate-related consequences auditors are likely to face upon reporting errors, they are unlikely to do so. The net 
result is that the effect of error-management climate on reporting willingness will be stronger for a mechanical error than a conceptual error. This leads to the following interaction hypothesis (see also Figure 1, Panel A):

H2: The difference in auditors' willingness to report post-review discovered errors in a open versus a blame organizational error-management climate will be greater for a mechanical error than for a conceptual error.

$$
<<<\text { Insert Figure } 1 \text { about here }>>>
$$

\section{Error Originator}

We suggest that auditors may engage in differential reporting behaviors upon detecting their own versus peers" errors. In particular, norms against "tattle tales" and "snitches" (e.g., Pershing 2003) suggest that auditors would be more likely to report their own errors than those of their peers. Indeed, the "whistle-blowing" research literature has often focused on determining why some people will report peer errors and mistakes, whether made deliberately or not, while others stand by and do nothing (e.g., Henik 2008; MacNab and Worthley 2008) as well as ways to encourage observers of such errors or mistakes to report them (e.g., Miceli et al. 2008). Indeed, the goal of this literature is to overcome the common response to stand by and do nothing when one observes others committing bad acts (Miceli and Near 1992). Given these social norms against "tattling," our third hypothesis is, ceteris paribus:

H3: Auditors are more willing to report post-review detected errors they committed than errors committed by their peers.

\section{Interaction between Error Type and Error Originator}

We now consider the originator of the error in light of error type (mechanical versus conceptual). First, we suggest that the earlier discussed competence attribution argument may be salient only when asking auditors about their own errors, as opposed to a peer auditor's errors, as most people are not focused on maintaining the reputation of their peer's competence as much as they 
are for their own. Indeed, the voluminous research literature (e.g., Pronin et al. 2004; Harvey and Weary 1984) on the "fundamental attribution error" has at its core the notion that people concentrate on attributing negative acts they commit to environmental causes (i.e. nature, superiors, co-workers, "the system") hence avoiding the attribution that their (lack of) competence caused the negative act to occur (Ross 1977). Hence, we expect that auditors are more willing to report self-committed mechanical errors (errors that are easier to excuse away as anyone could commit them) than conceptual errors (which reveals information about the auditor's competence).

On the other hand, when it comes to errors committed by a peer, we predict the opposite effect of error type. The whistle-blowing literature consistently finds that more severe errors committed by other organization members (i.e., peers) are associated with greater likelihood of being reported by the potential whistle blower (e.g., Vadera et al. 2009). To the extent that conceptual errors are viewed as more severe errors than mechanical errors (i.e., consistent with the competence attribution argument); the whistle-blowing literature would predict a greater likelihood of reporting conceptual errors than mechanical errors. Hence, we predict the following interaction, ceteris paribus:

H4: Auditors' willingness to report post-review discovered peer errors is greater for conceptual errors than mechanical errors and is greater for self-committed mechanical errors than self committed conceptual errors.

See Figure 2, Panel A for an illustration of the predicted interaction.

$$
<<<\text { Insert Figure } 2 \text { about here }>>>
$$

\section{Interaction between Error-Management Climate and Error Originator}

Finally, we predict that the effect of error-management climate will be greater for peercommitted errors than self-committed errors, as the whistle-blowing research has shown a 
greater likelihood of reporting others' errors when there is a positive organizational climate: "[...] research shows that individuals in organizations with team or friendship climates, strong ethical climates, or democratic climates are more likely to engage in whistle-blowing when they observe a wrongdoing" (Vadera et al. 2009: 583). Given that the open climate is closer to the description of the climates that encourage whistle-blowing whereas the sanction-oriented blame climate is not, we expect a relatively strong effect of error-management climate. Combining persistent social norms against "tattling" with a blame-oriented climate is expected to result in a significant resistance to reporting peer-committed errors. On the other hand, auditors are more likely to report self-committed errors than peer-committed errors to begin with (see H3), so the encouraging effect of error-management climate is expected to be relatively weaker. Hence, we predict the following interaction, ceteris paribus:

H5: The difference in auditors' willingness to report post-review discovered errors in an "open" versus a "blame" organizational error management climate will be greater for peer errors than for own errors.

See Figure 3, Panel A for an illustration of the predicted interaction.

$$
<<<\text { Insert Figure } 3 \text { about here }>>>
$$

\section{EXPERIMENT}

\section{Research Method}

\section{Design, Task and Procedure}

We designed and administered our experiment by using a two (error-management climate: blame vs. open) by two (error type: mechanical vs. conceptual) by two (error originator: self vs. peer) full-factorial design. Participants were randomly assigned to one of the eight treatment conditions. Materials were provided to the participants in a paper-based format. ${ }^{6}$

\footnotetext{
${ }^{6}$ All experiments were administered in Germany by a native German speaking faculty member. The faculty member adapted the case as necessary for the German audit environment and then translated the English case into German. A
} 
The case used in the current study is an adaptation (with permission) of the Burgstahler et al. (2000) case. We employed an actual example drawn from PCAOB reports (2004) which discussed an issue about sampling errors that was highly similar to the case we were adapting. In the actual case, the PCAOB inspection report faulted the audit firm for not projecting the sample to the population. This error could be either mechanical (i.e., the correct arithmetic projection was not done) or conceptual (i.e., no projection at all was done). Hence this inventory projection error allowed for great experimental control, as we did not have to use two different error scenarios in our case to deal with conceptual and mechanical errors.

Each participant received a packet of case materials, which included a brief introduction to the research project. The materials included (1) background information about the hypothetical audit engagement and client that included the first description of the errormanagement climate manipulation (open versus blame), (2) a series of working paper extracts including the error type manipulation (mechanical versus conceptual), (3) the dependent variables measurement, (4) manipulation checks and (5) a debriefing questionnaire.

Participants read a case related to an audit carried out by a large international audit firm. In the case the participants learned that a series of working papers related to the "Summary of Non-Booked Audit Differences (SNBAD)" had been detached from their main file during the review process, and the task was to "tidy up" the file for final review by the quality assurance partner before the audit report would be signed and the financial statements released.

professional translator (recommended by the Head of a major German research library) independently translated the case materials and the two reconciled any differences, which were minimal and almost exclusively focused on technical accounting and auditing terms. Further, four German-speaking business professionals read the case for general understandability and clarity. Next, the first audit firm that agreed to participate read and commented on the case prior to a pre-test. The case was then pre-tested on 15 auditors and modifications were made based on that pretest to further enhance understandability of the case materials. None of the pretest auditors took part in the actual experiment. 
The background information described the audit of a medium-size privately held manufacturing firm with debt agreements that require audited financial statements. ${ }^{7}$ In the planning phase of the audit, inherent risk, control risk and risk of material misstatement were assessed as moderate and materiality was set at $€ 1,000,000$ (equal to $5 \%$ of earnings before taxes). However, the audit plan specified an approach that relies on substantive testing and that all proposed or potential adjustments based on known errors in excess of $€ 50,000$, as well as all results of audit sampling, are posted to the SNBAD. The materials also stated that in prior years, management had readily made required adjustments. However, this year, without prior consultation with the audit firm, the client's president has provided shareholders and creditors with preliminary unaudited earnings information and strongly prefers to minimize adjustments to the financial statements because he believes that "such adjustments will unduly reduce shareholder and creditor confidence in management." Hence, unless absolutely necessary, he has asked the audit firm to propose no adjustments. Consequently, the office-managing partner of the audit firm has also made clear that only essential and minimal adjustments should be proposed to client management.

Following the error-management climate manipulation, participants were asked to consider three working papers that had previously been detached from the file in light of the task to reattach them to the correct place in the file to get it ready for a final quality review. The first working paper was the SNBAD, which summarized a number of adjustments leading to a total proposed adjustment. The remaining two working papers were extracts from the lead sheets, providing the details for the adjustment and its calculations. Through the hypothetical tidying up of the file, participants were made aware of an error in the adjustment (manipulated as either

\footnotetext{
${ }^{7}$ This is in addition to the general requirement in Germany that companies have what is referred to as a statutory audit in the Anglo-Saxon world.
} 
conceptual or mechanical) that had been made during working paper preparation. This resulted in discovering additional income reducing adjustments that needed to be added to the previously known overstatement. The combined overstatement of income totaled $€ 998,000$, which is slightly below materiality of $€ 1,000,000 .^{8}$

After examining the background information and the working papers, participants were asked to indicate their willingness to report the problem they had found (i.e., the additional overstatements) to an appropriate responsible person for the audit engagement (i.e., audit team leader, engagement manager, responsible partner, or quality reviewer). Finally, participants responded to a number of manipulation checks, debriefing questions, covariate measurements, demographic questions, and questions related to the understanding of the experimental case.

\section{Experimental Treatments}

Error-Management Climate: Following the client description and under the header "Office climate at your firm", the error-management climate was manipulated as either 'blame' or 'open'. We followed the underlying psychology and limited accounting research (e.g., Van Dyck et al. 2005; Gronewold and Donle 2010) to identify the factors to vary between the two types of office climate. These included open discussion of errors with others, obtaining others' help after discovering errors, thorough analysis of errors and their potential causes, and concern with getting errors corrected in addition to concerns about sanctions for being forthcoming about errors discovered. See Table 1 for the manipulation of error-management climate.

$$
<<<\text { Insert Table } 1 \text { about here }>>>
$$

\footnotetext{
${ }^{8}$ Note there was no sampling risk provided to the participants in either condition and if participants had considered a reasonable allowance for sampling risk, the total misstatement on the SNBAD would be nearing $€ 1.3$ million per our computations. We attempted to incorporate sampling risk into the experiment via means of a within-subjects design following Burgstahler et al. (2000), where after the first set of dependent variables are collected, we gave participants information about the missing consideration of sampling risk (i.e. a second conceptual error). However, due to unanticipated ceiling effects on the dependent variable in response to our first manipulations, virtually everyone indicated a very high willingness to report after considering the additional $€ 300,000$ in sampling risk, hence there was no significant variation across participants and experimental conditions.
} 
Error Type: The working papers that participants reviewed each contain a summation of the errors that were detected during the audit, posted to the SNBAD, and thus affected the client's total earnings (as a net overstatement of earnings). All "mechanical error" participants were told of the incorrect arithmetic in the projection to the population and provided with the correct computation, whereas in the "conceptual error" condition participants were told about the absence of the projection to the population and given the correct one. ${ }^{9}$

Error originator: The error originator manipulation featured language that focused on either the reader discovering his/her own errors (e.g., "you", "your", etc.) or a named peer described as at the same level in the firm as the reader (“Andreas Meyer"). ${ }^{10}$ This identification of error originator was introduced during the description of the audit engagement (e.g., noting it was Andreas Meyer or the reader him/herself ("you") who had prepared the working papers) and reinforced throughout the experimental materials. Participants in the "self-committed error" treatment were informed that they and their audit team had completed all planned fieldwork and that they themselves had prepared all working papers that were referred to in the materials. Hence, they had committed the errors in the adjustments proposed in the working papers. In the "peer error" condition, another auditor working at the same local office and member of the audit team was described as having prepared the working papers in which the errors were detected.

\section{Dependent Variable}

Participants responded on a scale from 0 (Very Unlikely) to 10 (Very Likely) to the following question: "How likely are you as an auditor at Good and Better WPG" to report the problem you

\footnotetext{
${ }^{9}$ Implicitly in both conditions, the conceptual error of not allowing for sampling risk was not brought to the attention of the auditors. To the extent that participants consider the implicit conceptual error with respect to sampling risk, we bias against finding our expected results.

${ }^{10}$ The use of a first name (instead of only the surname) in Germany supports the notion that it is a peer relationship and hence we mention the first name consistently to reinforce the manipulation that it was a peer whose error the auditor discovered.

${ }^{11}$ Good and Better WPG is the name of the fictitious audit firm in our experiment.
} 
found in the finished goods working paper to an appropriate responsible person for this audit engagement at Good and Better (e.g., audit team leader, engagement manager, responsible partner, or quality reviewer)?" This willingness to report measure reflects the emphasis on the individual auditor's responsibility in the case.

\section{Sample}

198 German auditors from a Big-4 audit firm participated in the experiment administered during firm training. ${ }^{12}$ Four participants were eliminated as outliers (i.e., responses more than 4 standard deviations from the mean). Four participants had missing values for the dependent variable, and hence were removed. Two sessions were administered in two different German locations. At least one of the researchers was present at all experimental sessions to ensure that the participants followed the experimental instructions and worked quietly and independently. Participants have sufficient audit experience (mean equals 10.47 months served on audit engagements) to carry out the task per their firm. Further, as desired, the participants had limited experience with reviewing activities (mean equals 1.2 months). See Table 2 for more demographic details about the participants. ${ }^{13}$

Self-reported experience with reviewer activities (measured in months) varies significantly across experimental conditions $(\mathrm{p}<0.05)$ and is correlated with the dependent variable; hence, we include it as a control variable. ${ }^{14}$ On a scale of -5 through +5 , we asked a

\footnotetext{
${ }^{12}$ In addition, as reported in the robustness of results section following the results, we conducted a large scale pilot test of the experiment with a sample of 176 auditors from two other Big 4 firms and three regional firms. Based on the insights from this pilot test, final modifications were made to the case materials for our actual experiment. None of the pre-test participants took part in the pilot test and none of the pilot test participants took part in the experiment we report.

${ }^{13}$ To verify whether randomization across experimental treatments was successful, we examined whether the following demographic factors vary across treatment conditions: age, gender, auditor rank, academic degree, audit engagement experience, and experience with similar errors like those in the case. None of these items are significantly different across treatment conditions (all p's $>0.24$ ).

${ }^{14}$ Ten participants had missing values for review experience. For these missing values we entered the variable's mean or zero months, the most common level of experience. Choice of missing value substitution makes no
} 
number of questions to verify participants' recall and understanding of some important case details. On average, they recalled correctly that (1) in prior year audits, the client had always readily booked audit proposed adjustments (mean equals 2.90), (2) management is currently strongly committed to reporting the net income figure as is, unless the audit firm threatens to qualify the report (mean equals 2.40), and (3) adding up the originally known errors, the detected errors and the omission for sampling risk renders the total error above materiality (mean equals 3.22). Participants also recalled that the potential adjustments recorded in the SNBAD prior to the current error being discovered were below (rather than marginally below, following the error in the working paper detection) the materiality threshold, as evidenced by a negative mean response of -2.55 (scale anchored at -5 ). Overall, these responses indicate that participants understood the case.

$<<<$ Insert Table 2 about here $>>>$

\section{Results}

\section{Manipulation Checks}

Employing multiple dimensions of error-management climate, we verified the effectiveness of this manipulation. ${ }^{15}$ On a scale from "Strongly Disagree" (-5) through "Strongly Agree" (+5), participants in the 'open climate' treatment significantly agreed, as expected, more strongly with each of the following assertions than participants in the 'blame climate' treatment (all p's $<0.001)$ :

difference in our analyses and leaving the review experience control variable out of the analyses only strengthens our results. Two additional potential control variables were identified as varying across experimental treatments: self-reported error projection experience on actual audits and self-reported participants' own error management climate experience. Neither of these variables were significant in our analysis $(p>0.10)$ and hence are not included in the results reported.

${ }^{15}$ The seven questions are based on items from the underlying psychology literature on error-management climate (e.g., Van Dyck et al. 2005). Three questions were about climate issues directly manipulated in the case description and four questions are about broader inferences about error management climate that are not found in a 1:1 fashion. Those questions were taken from the "organizational error culture" scale developed by Van Dyck et al. (2005), which has already been successfully tested in an auditing setting, too (Gronewold and Donle 2010). 
(1) "At this office of Good and Better WPG, a person that makes an error will receive full support by others" (1.47 vs. -1.45);

(2) "At this office of Good and Better WPG, there is a climate characterized by "being open for improvement" in the case of errors that have occurred in carrying out audit procedures" (2.69 vs. -1.60$)$;

(3) "At this office of Good and Better WPG, problems are openly discussed" (2.28 vs. -1.51$)$; and

(4) "At this office of Good and Better WPG, when someone makes an error, he/she shares it with others so that they won't make the same mistake" (1.90 vs. 0.26$)$;

(5) "At this office of Good and Better WPG, in general people warn each other about problems that might come up" (1.15 vs. - 0.47$)$.

Participants in the 'open climate' treatment also agreed significantly less with the following two assertions than participants in the 'blame climate' treatment (all p's $<0.001)$ :

(6) "At this office of Good and Better WPG, there is a climate characterized by "getting it right the first time" in the case of errors that have occurred in carrying out audit procedures" (-0.73 vs. 1.79$)$; and

(7) "At this office of Good and Better WPG, auditors who make errors in carrying out audit procedures or who miss errors in reviewing work results will likely face negative consequences at desired changes on a transfer basis to another office within the audit firm, even if the errors occurred for the first time" (-3.03 vs. 3.03).

Thus, we conclude that the manipulation of the error-management climate was successful.

To test for understanding of the error type manipulation (using the same $-5 /+5$ scale as before) participants indicated their agreement with the statement "The detected error in the work papers was more like a 'mechanical' error (e.g. calculation error, typing error, writing error, or the like, in audit or evaluation steps carried out) than a 'conceptual' error (e.g. missing of a complete audit step or of a crucial evaluation step, a fundamental thinking error, or the like)". The mean level of agreement for the mechanical error condition (1.82) was significantly greater $(p<0.001)$ than for the conceptual error $(-1.42)$. This suggests an effective manipulation of error type.

To test for understanding of the error originator manipulation, using again the "Strongly Disagree" $(-5)$ to "Strongly Agree" $(+5)$ scale, participants indicated if they themselves had 
prepared the working papers on which the errors were detected. The mean response was 4.02 for the 'own error' treatment and -3.34 for the 'peer's error' treatment. The two means are significantly different $(\mathrm{p}<0.001)$, suggesting a successful manipulation of error originator.

Finally, Table 3 reports the overall results of the experiment, including raw descriptive statistics for the dependent variables (Panel A) and the ANCOVA analysis (Panel B). The dependent variable is how likely the participants themselves would be to report the error. ${ }^{16}$ Table 3 Panel B's ANCOVA shows the overall model is highly significant at conventional levels $(\mathrm{F}(1,181)=3.30, \mathrm{p}<0.002)$. Further, as expected, the three-way interaction is not significant $(\mathrm{p}>0.30)$.

$<<<$ Insert Table 5 about here $>>>$

\section{Hypothesis Testing}

We now turn to testing our five hypotheses. Where directional predictions are made we conduct one-tailed tests as noted in the text. We buffer for multiple comparisons, where appropriate, in analyzing the simple effects. All tests of mean differences are for least square adjusted means (i.e., adjusted for the covariate months of review experience). No differences are found if raw mean tests are carried out although significance levels are generally higher.

Error management hypothesis and interaction with error type: The main effect of errormanagement climate is significant $\left(\mu_{1}-\mu_{2}=8.78-8.33=0.45, \mathrm{t}=1.86, \mathrm{p}<0.03\right.$, one-tailed $)$, supporting H1. However, this is qualified by the interaction of climate with error type being significant $(\mathrm{F}(1,181)=4.38, \mathrm{p}<0.04)$. Examining the form of the significant interaction between error-management climate and error type (see Figure 1, Panel B), we find, as predicted in H2

\footnotetext{
${ }^{16}$ To check for potential social desirability issues about self reporting errors (e.g., Clement and Krueger 2000; Mikulincer and Horesh 1999; Ruvolo and Fabin 1999; Smith 1997) we also asked how their peers would respond to the same question. We found no significant differences between the responses for their own willingness to report the error versus their perceptions of their peer's willingness to report the same error.
} 
(Figure 1 Panel A), the willingness to report mechanical errors in the open climate is greater than in the blame climate $\left(\mu_{1}-\mu_{2}=9.09-8.14=0.95, \mathrm{t}=2.78, \mathrm{p}<0.01\right.$, one-tailed $)$. Further, there is no difference in the reporting likelihood for conceptual errors being reported between the blame and open climate conditions $\left(\mu_{1}-\mu_{2}=8.48-8.53=-0.05\right.$, n.s. $)$. Hence, consistent with $H 2$, we find that there is a greater difference in willingness to report mechanical errors in the open condition whereas that difference is less (in this case non-existent) in the conceptual error condition. Error originator hypotheses and interactions with error type and error climate: $\mathrm{H} 3, \mathrm{H} 4$ and $\mathrm{H} 5$ examine the effect of error originator on willingness to report errors including its interaction with error climate and error type. The main effect of error originator predicted in $\mathrm{H} 3$, that own errors are more likely to be reported than other's errors, is directionally correct $\left(\mu_{1}-\mu_{2}=8.73-8.39=0.34\right)$, but is only marginally significant in a one-tailed test $(\mathrm{t}=1.43, \mathrm{p}<0.08)$. However, as error originator is involved in two significant interactions, the simple effects within each of those significant interactions must be examined to determine if there are qualifications to this marginally significant main effect.

First, we examine the interaction of originator with error type via simple effects analysis. In the mechanical error condition, self-committed errors are more likely to be reported than peercommitted errors $\left(\mu_{1}-\mu_{2}=9.09-8.14=0.95, \mathrm{p}<0.04\right)$, whereas in the conceptual error condition, own errors are equally as likely to be reported as peer errors $\left(\mu_{1}-\mu_{2}=8.37-8.64=-0.27\right.$, n.s. $)$. Second, we examine the interaction of originator with error management climate via simple effects analysis. Here we find that auditors are more likely to report self-committed errors than peer's errors in the blame climate $\left(\mu_{1}-\mu_{2}=8.80-7.86=0.94, \mathrm{t}=2.75, \mathrm{p}<0.01\right)$, whereas there is no difference in an open climate $\left(\mu_{1}-\mu_{2}=8.65-8.91=-0.26, t=-0.77\right.$, n.s. $)$. Hence, we find that H3's predicted effect, that there is a greater willingness to report own versus peer errors, holds for 
mechanical errors but not for conceptual errors, and in the blame climate but not in the open climate.

$\mathrm{H} 4$ predicts that auditors are more willing to report a mechanical than a conceptual error when the error was committed by themselves, but that this error type effect reverses when a peer committed the error. Error originator is significant in the interaction with error type $(F(1,181)=$ 6.36, $\mathrm{p}<0.02$ ) (see Table 3, Panel B) and the form is as predicted by H4 (see Figure 2 Panel A versus Panel B). In the self-committed error condition, as predicted, mechanical errors are more likely to be reported than conceptual errors $\left(\mu_{1}-\mu_{2}=9.09-8.37=0.72, t=2.13, p<0.03\right.$ one-tailed). In the peer-committed condition, the difference reverses as predicted $\left(\mu_{1}-\mu_{2}=8.14-8.64=-0.50\right)$ but not significantly ( $\mathrm{t}=-1.43$, n.s.). Hence, our results partially support $\mathrm{H} 4$ in that the directional effects reverse as predicted, but only one of the differences is significant.

$\mathrm{H} 5$ predicts that the effect of error-management climate will be greater for peercommitted errors than for self-committed errors. Error originator is significant in the interaction with error-management climate $(F(1,181)=6.26, \mathrm{p}<0.02)$, as reported in Table 3 Panel B. An examination of the simple effects of error-management climate in the two error originator conditions (see Figure 3 Panel B) reveals that auditors are more likely to report peer-committed errors in an open climate, as compared to a blame climate $\left(\mu_{1}-\mu_{2}=8.91-7.86=1.05, \mathrm{t}=3.04\right.$, $p<0.01$, one-tailed), but when they themselves committed the error, the effect of climate is not significant $\left(\mu_{1}-\mu_{2}=8.65-8.80=-0.15\right.$, n.s. $)$. Hence, the open error management climate will result in more peer errors being reported than a blame management error climate. However, instead of a weaker but positive effect we expected in the self-committed error condition for reporting errors in an open climate, the effect of climate was not significant for self-committed errors being reported. It should be noted that the final level of reporting for peer errors in the open error 
management climate is not significantly different from either of the self error conditions (8.91 versus 8.65 and 8.80 , n.s. for both), hence the effect of open error management climate is to increase the likelihood of reporting peer errors to the same level as reporting own errors in both error climates. Thus, overall there is partial support for $\mathrm{H} 5$.

\section{Potential ceiling effects and robustness of experimental findings}

The grand mean for the dependent variable in the experiment was 8.57 (see Table 3 Panel A) with roughly $33 \%$ of respondents at the top value of the scale " 10 ". This may indicate the presence of "ceiling effects" (too high of a percentage of the data is clustered at the top of the scale). However, most research on ceiling effects only discusses problems with much higher percentages clustered at one end of the scale (e.g., Uttl 2005). To deal with this issue and to provide some evidence on the robustness of our error management climate hypothesis we discuss the results of our pilot test.

In our pilot test we examined a two (error-management climate: blame vs. open) by two (error type: mechanical+conceptual vs. conceptual+conceptual) with a sample of 176 auditors. ${ }^{17}$ The error management climate manipulation was the same as that used in this study. The error type manipulation featured a mechanical and a conceptual error similar to those used in this experiment but both conditions also featured a second conceptual error. That second conceptual error was in both conditions that sampling risk had been ignored and this was made explicit as part of the discovered error analysis information presented to each participant.

This experimental design allows us to examine the effects of $\mathrm{H} 1$, the error management climate hypothesis, but under different experimental design conditions. Specifically, design differences included making the initial SNBADs show lower amounts of proposed adjustments

\footnotetext{
${ }^{17} 136(77.3 \%)$ came from two Big-4 firms (which differed from the one in the actual experiment) and $40(22.7 \%)$ from three regional audit firms.
} 
(approximately $€ 390,000$ ) in the pilot test instead of the higher initial amounts (approximately $€ 660,000)$ in this experiment. In addition, the pilot test explicitly stated that sampling risk was approximately $€ 300,000$, adding the second conceptual error to each of the mechanical and conceptual error conditions. Total overstatements after error discovery were slightly above materiality in the pilot test (i.e., total misstatement at $€ 1,020,000$ ), which differed from slightly below materiality $€ 998,000$ in the experiment (however, note that in the experiment sampling risk was not explicitly mentioned before our dependent variable was collected). In both the pilot test and the current experiment materiality was $€ 1,000,000$. All manipulation checks and tests of experimental understandability worked as they did in the experiment.

Following the Burgstahler et al. (2000) findings that auditors tend to anchor on known errors (i.e. differences in our study) when recommending adjustments, we expected that the smaller amount of known differences in the pilot test (approximately $€ 390,000)$ than in the experiment (approximately $€ 660,000$ ) would result in a lower overall mean for the dependent variable (i.e. willingness to report) in the pilot test than in the final experiment. This should occur because in the experiment the known errors are closer to materiality than in the pilot test, hence more likely need to be dealt with by reporting them to appropriate parties. As in the main experiment all reported means are least squared adjusted with one-tailed tests given directional predictions and buffered for multiple comparisons where appropriate.

The grand mean for error reporting willingness in the experiment $\left(\mu_{1}=8.57\right)$ is significantly greater than the grand mean in the pilot test $\left(\mu_{2}=6.35\right)$ by $2.22(t=9.36, p<0.001)$. Relative to the experiment, the pilot test only features $6.8 \%$ (versus 33\%) of the data at the maximum scale value of 10 . Hence, in the pilot test we have less concern about possible ceiling effects than in the experiment. 
Results from the pilot test ANCOVA show a highly significant main effect for error management climate $(F(1,171)=5.74, \mathrm{p}<0.001)$. Consistent with the expectation that open error management climate will result in greater willingness to report than a blame climate, we find a highly significant main effect $\left(\mu_{1}-\mu_{2}=7.01-5.62=1.39, \mathrm{t}=2.40, \mathrm{p}<0.001\right)$. However, there was also a marginally significant interaction of error climate with error type $(F(1,171)=2.86, p<0.10)$, therefore this main effect needs to be examined in light of simple effects analysis.

In the simple effects analysis, we find that the conceptual error difference in willingness to report between the open and blame climate is $2.14\left(\mu_{1}-\mu_{2}=7.42-5.28=2.14\right)$, which is highly significant $(\mathrm{t}=3.82, \mathrm{p}<0.001$, one-tailed). In addition we find that the willingness to report errors discovered difference for mechanical errors in the open versus blame condition is smaller than for conceptual errors at $0.82\left(\mu_{1}-\mu_{2}=6.78-5.96=0.82\right)$, but still marginally significant $(\mathrm{t}=1.50$, $\mathrm{p}<0.07$, one-tailed). Thus, both simple effects analyses show the same pattern as predicted and found in $\mathrm{H} 1$ of our current experiment, that open error climate leads to a greater likelihood of reporting errors.

Overall, our pilot test results provide some confidence that ceiling effects did not affect our reported experimental results. Further, the results from the pilot test show that the effects of error-management climate are robust to different specifications of parameters in our case design. Hence, the pilot test can be seen as providing a robustness check for our experiment on at least two dimensions.

\section{DISCUSSION}

In this study we identify three factors that may affect the willingness to report discovered errors: the organization's error-management climate, the type of audit error and whether the error is made by the auditor or by a peer. We focus on errors detected by auditors that are suggestive of a 
material misstatement in the client's financial statements discovered after all detailed file review has been completed but before the financial statements have been released, hence the errors are potentially correctible. We provide evidence that an open error-management climate leads to greater auditor's willingness to report the post-review discovered error than a blame climate does, but only when the error is mechanical (rather than conceptual) and when the error was committed by a peer (rather than one-self). Further, we found that who committed the error mattered in that auditors were more willing to report their own errors, especially mechanical errors, in the blame condition whereas they were less willing to report peer errors, especially if they were mechanical, in the same condition. Interestingly, own errors were highly likely to be reported, regardless of error-management climate and error type and were equal to the reporting of peers' conceptual errors in an open climate. Overall, the evidence is compelling that errormanagement climate and who committed the original error affects the willingness of auditors to report post-review discovered working paper errors and that error type has situation-specific effects on willingness to report errors.

Some limitations to this study beyond those inherent with any experimental research should be mentioned. First, as the experiment is carried out in Germany (and in German) with German auditors, some may argue that this limits generalizability. However, as experiments generalize via theory (Campbell and Stanley (1963) is a definitive reference work in this area) there is no reason to believe that results would not generalize to other countries unless there are significant psychological differences that would interact with our manipulations that we are unaware of. Second, we chose a setting where the error was one that affected the substance of the financial statements (i.e., overstatement of income). Errors that involve the omission of major audit procedures without any evidence that their being carried out would reveal potentially 
material effects to the financial statements may be dealt with differently by auditors. However, to date the literature has not yet shown a difference between the two in other areas (i.e., reviewer detection of working paper errors). Third, it is possible that in some of our tests we experienced ceiling effects. However, that would bias against finding any significant results and we showed, using the pilot test data, that the results are robust to alternative specifications of case parameters for the error management climate conclusion.

Implications of this research for practice suggest that the office error-management climate is a significant factor in conducting high quality audits and that encouraging an open and accepting error management climate will result in a greater willingness by junior auditors to report errors they discover in the working papers. Further, this research shows that problems documented in the whistle-blowing literature about "not squealing" norms also affects auditors' willingness to report their peers' errors, at least for mechanical errors. Finally, we confirm the continued usefulness of the error type classification (i.e. mechanical vs. conceptual errors) as these two types of errors interacted in very different ways with the error-management climate and the error originator. 


\section{REFERENCES}

Alderman, C. W., and J. W. Deitrick. 1982. Auditors' perceptions of time budget pressures and premature sign-offs: A replication and extension. Auditing: A Journal of Practice \& Theory. 1(2): 54-68.

American Institute of Certified Public Accountants (AICPA). 2009. Statement on Quality Control Standard (SQCS) 10: A Firm's System of Quality Control.

Asare, S. K., and L. S. McDaniel. 1996. The effects of familiarity with the preparer and task complexity on the effectiveness of the audit review process. The Accounting Review. 71(2): $139-159$.

Bamber, E. M., and R. J. Ramsay. 1997. An investigation of the effects of specialization in audit workpaper review. Contemporary Accounting Research. 14(3): 501-513.

Bamber, E. M., and R. J. Ramsay. 2000. The effects of specialization in audit workpaper review on review efficiency and reviewers' confidence. Auditing: A Journal of Practice \& Theory. 19(2): 147-157.

Bowrin, A. R. 1998. Review and synthesis of audit structure literature. Journal of Accounting Literature. 17: 40-71.

Brazel, J.F., Agoglia, C.P., and R.C. Hatfield. 2004. Electronic versus face-to-face review: The effects of alternative forms of review on auditors' performance. The Accounting Review. 79(4): 949-966.

Burgstahler, D., S. M .Glover and J. Jiambalvo. 2000. Error projection and uncertainty in the evaluation of aggregate error. Auditing: A Journal of Practice \& Theory. 19(1): 79-99.

Campbell, D. T., and J. C. Stanley. 1963. Experimental and quasi-experimental designs for research. Boston, Houghton Mifflin Company.

Clement, R. W., and J. Krueger. 2000. The primacy of self-referent information in perceptions of social consensus. British Journal of Social Psychology. 39(2): 279-299.

Covaleski, M. A., M. W. Dirsmith, and J. B. Heian.1997. Structure and agency in an institutionalized setting: The application and social transformation of control in the Big Six. Accounting, Organizations and Society. 22(1): 1-28.

Covaleski, M. A., M. W. Dirsmith, J. B. Heian, and S. Samuel. 1998. The calculated and the avowed: Techniques of discipline and struggles over identity in Big Six public accounting firms. Administrative Science Quarterly. 43(2): 293-328.

Desmurget, M., and S. Grafton. 2000. Forward modeling allows feedback control for fast reaching movements. Trends in Cognitive Sciences. 4(11): 423-431. 
Dirsmith, . W. and M. A. Covaleski. 1985. Informal communications, nonformal communications and mentoring in public accounting firms. Accounting, Organizations and Society. 10(2): 149-170.

Edmondson, A. C. 1999. Psychological safety and learning behavior in work teams. Administrative Science Quarterly. 44(2): 350-383.

Ferguson, A., J. Francis, and D. Stokes. 2003. The effects of firm-wide and office-level industry expertise on audit pricing. The Accounting Review. 78(2): 429-448.

Francis, J., L. E. Maydew, and H. C. Sparks. 1999. The role of big 6 auditors in the credible reporting of accruals. Auditing: A Journal of Practice and Theory. 18(2): 17-34.

Ganushchak, L. Y., and N. O. Schiller. 2006. Effects of time pressure on verbal self-monitoring: An ERP study. Brain Research. 1125: 104-115.

Ganushchak, L. Y., and N. O. Schiller. 2008a. Motivation and semantic context affect brain error-monitoring activity: An event-related brain potentials study. NeuroImage. 39(1): 395405.

Ganushchak, L. Y., and N. O. Schiller. 2008b. Brain error-monitoring activity is affected by semantic relatedness: An event-related brain potentials study. Journal of Cognitive Neuroscience. 20(5): 927-940.

Gibbins, M. and J. Newton. 1994. An empirical exploration of complex accountability in public accounting. Journal of Accounting Research. 32(2): 165-186.

Gronewold U., and M. Donle. 2010. Audit firms' organizational error climate and auditors' predispositions to handling errors. Working Paper, Ruhr University Bochum.

Harding, N., and K. T. Trotman. 1999. Hierarchical differences in audit workpaper review performance. Contemporary Accounting Research. 16(4): 671-684.

Hartsuiker, R. J., and H. H. J. Kolk. 2001. Error monitoring in speech production: A computational test of the perceptual loop theory. Cognitive Psychology. 42(2): 113-157.

Harvey, J. H. and G. Weary. 1984. Current Issues in Attribution Theory and Research. Annual Review of Psychology. 35: 427-459.

Henik, E. 2008. Mad as hell or scared stiff? The effects of value conflict and emotions on potential whistle-blowers. Journal of Business Ethics. 80:111-119.

Hermanson, H. M. 1997. The effects of audit structure and experience on auditors' decisions to isolate errors. Behavioral Research in Accounting. 9(Supplement): 76-93.

Homsma, G. J., C. Van Dyck, D. De Gilder, P. L. Koopman, and T. Elfring. Learning from error: The influence of error incident characteristics. Journal of Business Research. 62(1): 115122. 
Hyatt, T. A., and D. F. Prawitt. 2001. Does congruence between audit structure and auditors' locus of control affect job performance? The Accounting Review. 76(2): 263-274.

International Auditing and Assurance Standards Board (IAASB). 2009. International Standard on Quality Control (ISQC) 1: Quality Control for Firms that Perform Audits and Reviews of Financial Statements, and Other Assurance and Related Services Engagements. International Federation of Accountants.

Kelley, T., and L. Margheim. 1990. The impact of time budget pressure, personality, and leadership variables on dysfunctional auditor behavior. Auditing: A Journal of Practice \& Theory. 9(2): 21-42.

Kennedy, J. 1993. Debiasing audit judgment with accountability: A framework and experimental results. Journal of Accounting Research. 31(2): 231-245.

Levelt, W. J. M. 1983. Monitoring and self-repair in speech. Cognition. 14(1): 41-104.

Levelt, W. J. M. 1989. Speaking: From intention to articulation. Cambridge, MA: MIT Press.

Luu, P., T. Flaisch, and D. M. Tucker. 2000. Medial frontal cortex in action monitoring. The Journal of Neuroscience. 20(1): 464-469.

MacNab, B. R., and Worthley, R. 2008. Self-Efficacy as an Intrapersonal Predictor for Internal Whistleblowing: A US and Canada Examination. Journal of Business Ethics. 79(4): 407421.

Malone, C. F., and R. W. Roberts. 1996. Factors associated with the incidence of reduced audit quality behaviors. Auditing: A Journal of Practice \& Theory. 15(2): 49-64.

McDaniel, L. S. 1990. The effects of time pressure and audit program structure on audit performance. Journal of Accounting Research. 28(2): 267-285.

McNair, C. J. 1991. Proper compromises: The management control dilemma in public accounting and its impact on auditor behavior. Accounting, Organizations and Society. 16(7): 635-653.

Miceli, M. P., and J. P. Near. 1992. Blowing the Whistle: The Organizational and Legal Implications for Companies and Employees. Lexington, MA: Lexington Books.

Miceli, M. P., J. P. Near, and T. M. Dworkin. 2008. Whistle-blowing in Organizations. Mahwah, NJ: Taylor and Francis, LEA Organization and Management Series.

Miceli, M. P., J. P. Near, and T. M. Dworkin. 2009. A word to the wise: how managers and policy-makers can encourage employees to report wrongdoing. Journal of Business Ethics. 86(3): 379-396. 
Mikulincer, M., and N. Horesh. 1999. Adult attachment style and the perception of others: The role of projective mechanisms. Journal of Personality and Social Psychology. 76(6): 10221034.

Nooteboom, S., and H. Quené. 2008. Self-monitoring and feedback: A new attempt to find the main cause of lexical bias in phonological speech errors. Journal of Memory and Language. 58(3): 837-861.

Owhoso, V. E., W. F. Messier, Jr., and J. G. Lynch, Jr. 2002. Error detection by industryspecialized teams during sequential audit review. Journal of Accounting Research. 40(3): 883-900.

Pershing, J. L. 2003. To snitch or not to snitch? Applying the concept of neutralization techniques to the enforcement of occupational misconduct. Sociological Perspectives. 46(2): 149-178.

Pierce, B., and B. Sweeney. 2006. Perceived adverse consequences of quality threatening behaviour in audit firms. International Journal of Auditing. 10(1): 19-39.

Postma, A. 2000. Detection of errors during speech production: A review of speech monitoring models. Cognition. 77: 97-131.

Pronin, E., T. Gilovich, and L. Ross 2004. Objectivity in the eye of the beholder: Divergent perceptions of bias in self versus others. Psychological Review. 111(3): 781-799.

Public Company Accounting Oversight Board (PCAOB). 2004. Report on the 2003 Limited Inspection of PricewaterhouseCoopers LLP. August 26.

Rabbitt, P. 1969. Psychological refractory delay and response-stimulus interval duration in serial, choice-response tasks. Acta Psychologica. 30: 195-219.

Rabbitt, P. 1990. Age, IQ and awareness, and recall of errors. Ergonomics. 33(10/11): 12911305.

Ramsay, R. J. 1994. Senior/manager differences in audit workpaper review performance. Journal of Accounting Research. 32(1): 127-135.

Reynolds, J. K. and J. Francis. 2000. Does size matter? The influence of large clients on officelevel auditor reporting decisions. Journal of Accounting and Economics. 30(3): 375-400.

Rodríguez-Fornells, A., A. R. Kurzbuch, and T. F. Münte. 2002. Time course of error detection and correction in humans: Neurophysiological evidence. The Journal of Neuroscience. 22: 9990-9996.

Ross, L. 1977. The intuitive psychologist and his shortcomings: Distortions in the attribution process. In Advances in Experimental Social Psychology (10), edited by L. Berkowitz, 173-220. New York, NY: Academic Press. 
Rothschild, J. and T. D Miethe. 1999. Whistle-blower disclosures and management retaliation: The battle to control information about organization corruption. Work and Occupations. 26(1): 107-128.

Ruvolo, A. P., and L. A. Fabin. 1999. Two of a kind: Perceptions of own and partner's attachment characteristics. Personal Relationships. 6(1):57-79.

Rybowiak, V., H. Garst, M. Frese, and B. Batinic. 1999. Error orientation questionnaire (EOQ): Reliability, validity, and different language equivalence. Journal of Organizational Behavior. 20(4): 527-547.

Schneider, W., and R. M. Shiffrin. 1977. Controlled and automatic human information processing: I. Detection, search, and attention. Psychological Review. 84(1): 1-66.

Shiffrin, R. M., and W. Schneider. 1977. Controlled and automatic human information processing: II. Perceptual learning, automatic attending, and a general theory. Psychological Review. 84(2): 127-190.

Smith, E. 1997. Private selves and shared meanings: Or forgive us for our projections as we forgive those who project into us. Psychodynamic Counseling. 3(2): 117-131.

Smith, J. F., and T. Kida. 1991. Heuristics and biases: Expertise and task realism in auditing. Psychological Bulletin. 109(3): 472-489.

Tan, H.-T., and K. T. Trotman. 2003. Reviewers' responses to anticipated stylization attempts by preparers of audit workpapers. The Accounting Review. 78(2): 581-604.

Uttl, B. 2005. Measurements of individual differences: Lessons from memory assessment in research and clinical practice. Psychological Science. 16(6): 460-467.

Vadera, A.K., R. V. Aguilera and B. B. Caza. 2009. Making sense of whistle-blowing's antecedents: Learning from research on identity and ethics programs. Business Ethics Quarterly. 19(4): 553-586.

Van Dyck, C. 1997. Error climate and management issues: Its links to organizational performance. In Managerial Behavior and Practices: European Research Issues, edited by R. Pepermans, A. Buelens, C. J. Vinkenburg, and P. G. W. Jensen, 107-116. Amersfoort, NL: Acco.

Van Dyck, C. 2009. The tragic 1996 Everest expedition: A tale of error culture. Netherlands Journal of Psychology. 65(1): 22-34.

Van Dyck, C., M. Frese, M. Baer, and S. Sonnentag. 2005. Organizational error management culture and its impact on performance: A two-study replication. Journal of Applied Psychology. 90(6): 1228-1240.

Van Leuvan, P., and M. C. Wang. 1997. An analysis of students' self-monitoring in first- and second-grade classrooms. The Journal of Educational Research. 90(3): 132-143. 
Willett, C., and M. Page. 1996. A survey of time budget pressure and irregular auditing practices among newly qualified UK chartered accountants. British Accounting Review. 28(2): 101120. 
Table 1: Error-Management Climate Manipulation

\section{First introduction of office error management climate manipulation:}

Blame: The overall climate of this office of your firm is noted for a "getting it right the first time" mentality that reflects the office managing partner's own beliefs and actions. Errors in carrying out audit procedures are seen as signs of incompetence and reviewers who miss errors are at risk of at least demotion back to being preparers. You have seen top seniors coming from review meetings with managers and partners - after discussions about errors in files - nearly reduced to tears. Performance evaluations document such problems, complicating possibilities for future promotion within the firm.

(Open): The overall climate of this office of your firm is noted for an "open for improvement" mentality that reflects the office managing partner's own beliefs and actions. Errors in carrying out audit procedures are seen as a natural part of learning and reviewers who miss errors are coached by more experienced reviewers so they do not miss similar problems in the future. You have seen top seniors coming from review meetings with managers and partners - after discussions about errors in files - with a renewed determination to work harder next time. Performance evaluations do not document such problems if the person does not repeat them or is making progress in addressing them, allowing possibilities for future promotion within the firm.

\section{Later in the case the error-management climate manipulation is reinforced using the following:}

Blame None of the reviewers had noted this error, so maybe it was not so obvious. In part you are relieved when you think that the error cannot be easily detected; because you are not certain you want to go through a detailed debriefing about how you could have been "so stupid" as to miss something this obvious - despite the fact that two levels of review had also missed it. One thing is for certain: if you report this, your annual performance evaluation is going to become a much more negative one.

(Open): None of the reviewers had noted this error, so maybe it was not so obvious. In part you are relieved when you think that the error cannot be easily detected as it will result in a great learning opportunity when you sit down with the reviewers to determine how to ensure that this type of error is not missed again - especially given the fact that two levels of review had also missed it. One thing is for certain: if you report this, your annual performance evaluation is not going to be affected unless you continue to make the same error, which certainly will not occur. 
Table 2: Sample Demographics

\begin{tabular}{|c|c|c|c|}
\hline Variable & $\mathrm{N}=190^{*}$ & & \\
\hline \multicolumn{4}{|l|}{ Gender } \\
\hline Male & $109(57.4 \%)$ & & \\
\hline Female & $70(36.8 \%)$ & & \\
\hline Missing & $11(5.8 \%)$ & & \\
\hline \multicolumn{4}{|l|}{ Rank } \\
\hline Assistant auditor & $176(92.6 \%)$ & & \\
\hline Senior-associate & $2(1.1 \%)$ & & \\
\hline Senior & $3(1.6 \%)$ & & \\
\hline Manager & $0(0 \%)$ & & \\
\hline Other & $1(0.5 \%)$ & & \\
\hline Missing & $8(4.2 \%)$ & & \\
\hline \multicolumn{4}{|l|}{ Academic degree } \\
\hline Bachelor & $23(12.1 \%)$ & & \\
\hline Master & $6(3.2 \%)$ & & \\
\hline German Diploma, Master or State Examination & $151(79.5 \%)$ & & \\
\hline $\mathrm{PhD}$ & $1(0.5 \%)$ & & \\
\hline Missing & $9(4.7 \%)$ & & \\
\hline Variable & Mean & SD & $\mathrm{N}=190 *$ \\
\hline Age & 27.58 & 2.15 & 181 \\
\hline Audit engagement experience (months) & 10.47 & 4.66 & 183 \\
\hline Reviewer experience & 1.20 & 3.14 & 180 \\
\hline Statistical audit sampling experience $(0=$ Never; $10=$ All clients $)$ & 6.14 & 3.05 & 187 \\
\hline
\end{tabular}

* Data is missing where $\mathrm{N}$ is less than 190. 
Table 3: Your Willingness to Report Error to an Appropriate Person

Panel A: Raw Means and Standard Deviations of Report Willingness ${ }^{\mathrm{a}}$ by Error-Management Climate $^{\mathrm{b}}$, Error Type ${ }^{\mathrm{c}}$, and Error Originator ${ }^{\mathrm{d}}$

\begin{tabular}{|c|c|c|c|c|c|}
\hline $\begin{array}{c}\text { Error- } \\
\text { Management } \\
\text { Climate }\end{array}$ & Error Type & $\begin{array}{c}\text { Error } \\
\text { Originator }\end{array}$ & Mean & SD & $\mathbf{n}$ \\
\hline \multirow[t]{9}{*}{ Blame } & Conceptual & Self & 8.85 & 1.06 & 25 \\
\hline & & Peer & 8.19 & 1.82 & 23 \\
\hline & & Overall & 8.53 & 1.50 & 48 \\
\hline & Mechanical & Self & 8.85 & 1.49 & 24 \\
\hline & & Peer & 7.51 & 2.77 & 22 \\
\hline & & Overall & 8.21 & 2.27 & 46 \\
\hline & Overall & Self & 8.85 & 1.27 & 49 \\
\hline & & Peer & 7.86 & 2.33 & 45 \\
\hline & & Overall & 8.37 & 1.91 & 94 \\
\hline \multirow[t]{9}{*}{ Open } & Conceptual & Self & 7.90 & 1.96 & 25 \\
\hline & & Peer & 8.96 & 1.28 & 23 \\
\hline & & Overall & 8.41 & 1.74 & 48 \\
\hline & Mechanical & Self & 9.39 & 0.75 & 24 \\
\hline & & Peer & 8.81 & 1.49 & 24 \\
\hline & & Overall & 9.10 & 1.20 & 48 \\
\hline & Overall & Self & 8.63 & 1.66 & 49 \\
\hline & & Peer & 8.89 & 1.37 & 47 \\
\hline & & Overall & 8.75 & 1.52 & 96 \\
\hline \multirow[t]{9}{*}{ Overall } & Conceptual & Self & 8.37 & 1.63 & 50 \\
\hline & & Peer & 8.58 & 1.61 & 46 \\
\hline & & Overall & 8.47 & 1.61 & 96 \\
\hline & Mechanical & Self & 9.12 & 1.20 & 48 \\
\hline & & Peer & 8.19 & 2.27 & 46 \\
\hline & & Overall & 8.66 & 1.85 & 94 \\
\hline & Overall & Self & 8.74 & 1.48 & 98 \\
\hline & & Peer & 8.38 & 1.96 & 92 \\
\hline & & Overall & 8.57 & 1.73 & 190 \\
\hline
\end{tabular}

Legend:

${ }^{a}$ Own Report Willingness: Response to the likelihood you would report the problem found in the finished goods working paper to an appropriate responsible person for this audit engagement at Good and Better (e.g. audit team leader, engagement manager, responsible partner, or quality reviewer). ( $0=$ Very unlikely; $10=$ Very likely $)$.

b Error-Management Climate: Client office is described as one with an "open" learning and supportive error-management climate versus a "blame" climate.

cError Type: The type of the error is "mechanical" (e.g., a mis-computed calculation) versus "conceptual" (e.g., a non-performed needed step).

${ }^{\mathrm{d}}$ Error Originator: The originator of the error is the auditor (self) or the auditor's peer (peer). 
Panel B: Results of a 2x2x2 ANCOVA of Error-Management Climate, Error Originator, and Error Type on Own Report Willingness, Controlling for Reviewer Experience

\begin{tabular}{lrrrrr}
\hline \multicolumn{1}{c}{ Source } & $\begin{array}{c}\text { Type III Sum } \\
\text { of Squares }\end{array}$ & \multicolumn{1}{c}{$\begin{array}{c}\text { Mean } \\
\text { df }\end{array}$} & $\begin{array}{c}\text { F- } \\
\text { Square }\end{array}$ & $\begin{array}{c}\text { p- } \\
\text { value }\end{array}$ & \begin{tabular}{l} 
value \\
\hline Model
\end{tabular} \\
Error-Management Climate $(E M C)$ & 72.3 & 8 & 9.04 & 3.30 & 0.002 \\
Error Type (ET) & 9.51 & 1 & 9.51 & 3.47 & 0.065 \\
Error Originator (EO) & 0.54 & 1 & 0.54 & 0.20 & 0.657 \\
EMC x ET & 5.54 & 1 & 5.54 & 2.02 & 0.156 \\
EMC x EO & $\mathbf{1 2 . 0 0}$ & $\mathbf{1}$ & $\mathbf{1 2 . 0 0}$ & $\mathbf{4 . 3 8}$ & $\mathbf{0 . 0 3 8}$ \\
ET x EO & $\mathbf{1 7 . 1 6}$ & $\mathbf{1}$ & $\mathbf{1 7 . 1 6}$ & $\mathbf{6 . 2 6}$ & $\mathbf{0 . 0 1 4}$ \\
EMC x ET x EO & $\mathbf{1 7 . 4 2}$ & $\mathbf{1}$ & $\mathbf{1 7 . 4 2}$ & $\mathbf{6 . 3 6}$ & $\mathbf{0 . 0 1 3}$ \\
Reviewer Experience & 2.69 & 1 & 2.69 & 0.98 & 0.323 \\
Error & 7.74 & 1 & 7.74 & 2.90 & 0.091 \\
\hline
\end{tabular}

Legend:

${ }^{\mathrm{e}}$ Reviewer Experience: Self-reported experience with reviewer activities measured in months. All other terms defined in Panel A. 


\section{Figure 1}

Panel A: Predicted Interaction Effect of Error-Management Climate x Error Type on Report Willingness (H2)

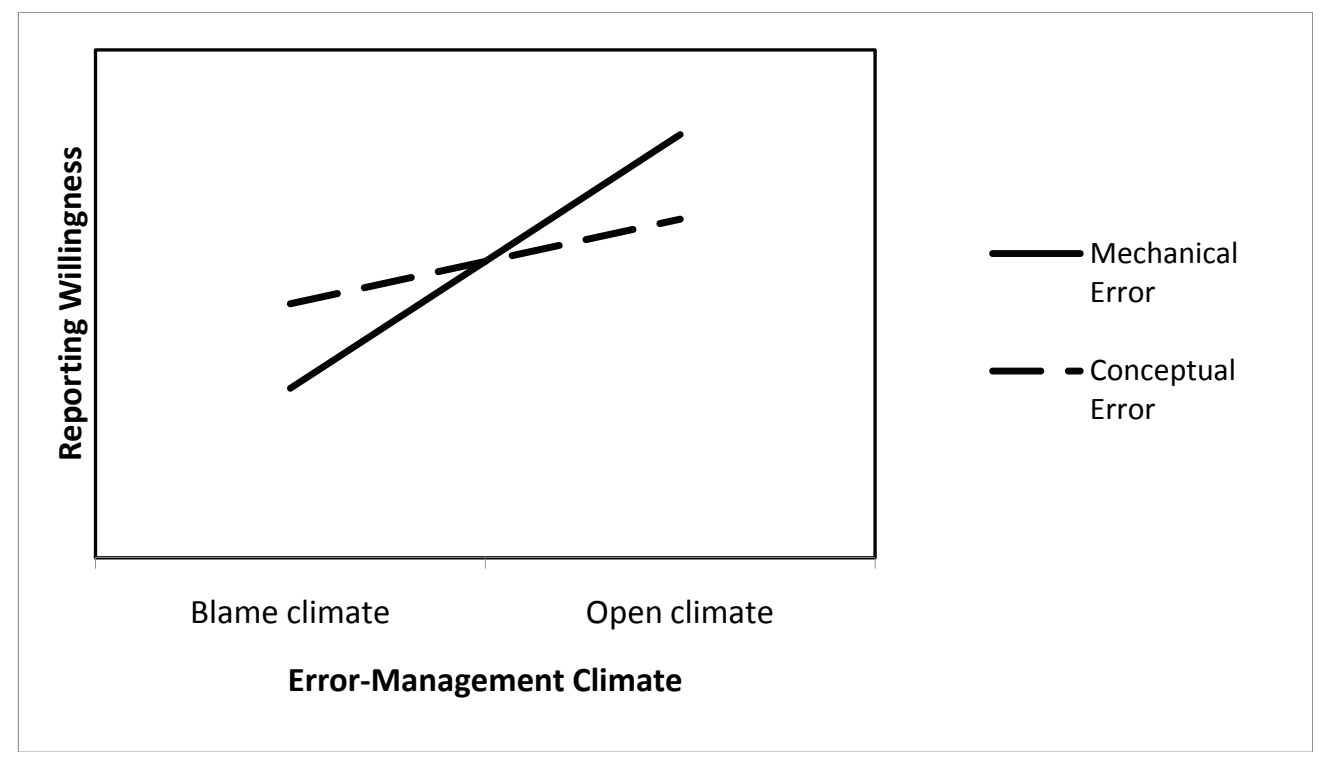

Panel B: Observed Interaction Plot: Error-Management Climate by Error Type on Report Willingness (Adjusted Means)

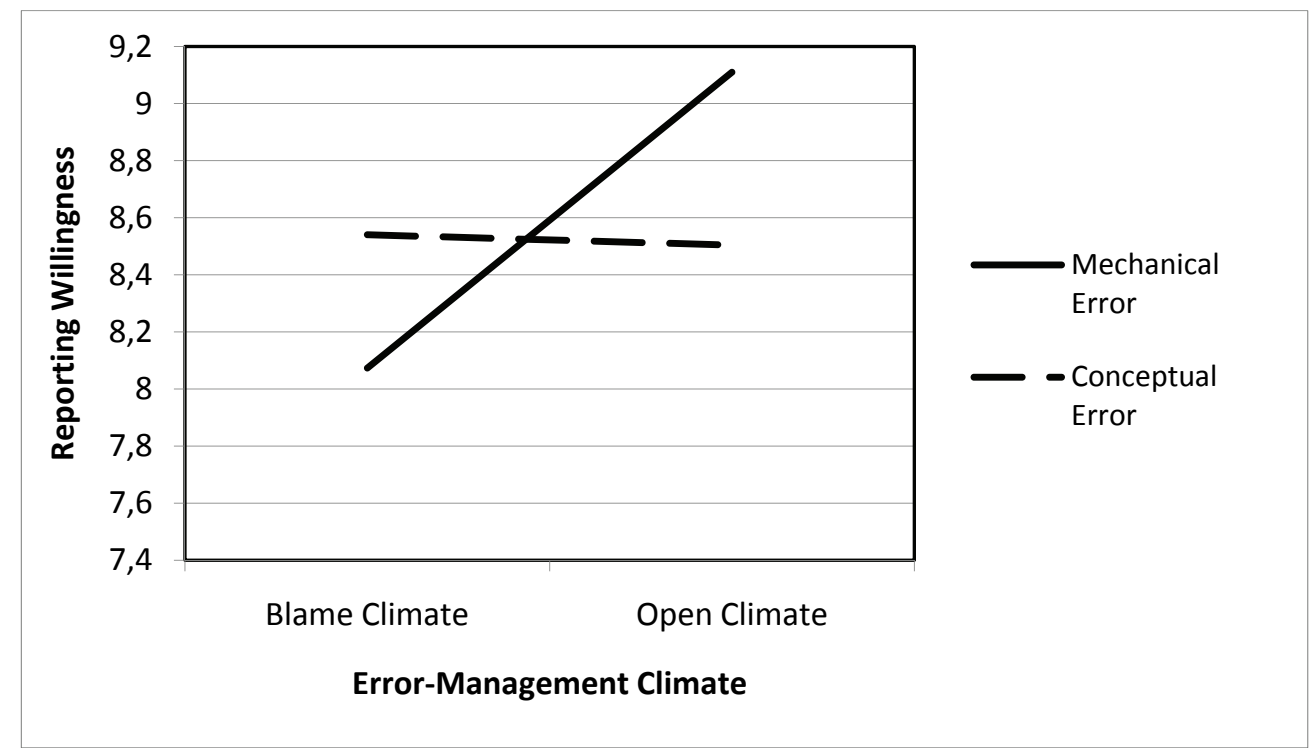




\section{Figure 2}

Panel A: Predicted Interaction Effect of Error Type x Error Originator on Report Willingness (H4)

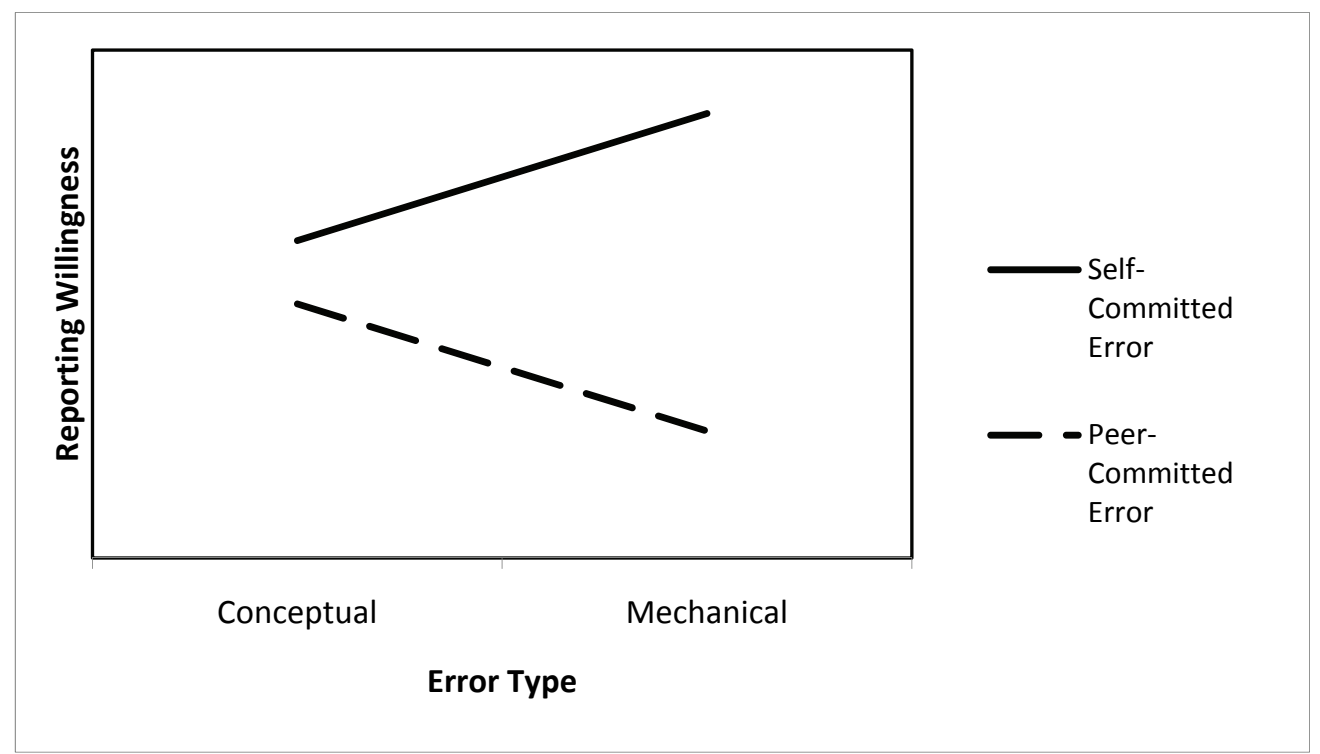

Panel B: Observed Interaction Plot: Error Originator by Error Type Report Willingness (Adjusted Means)

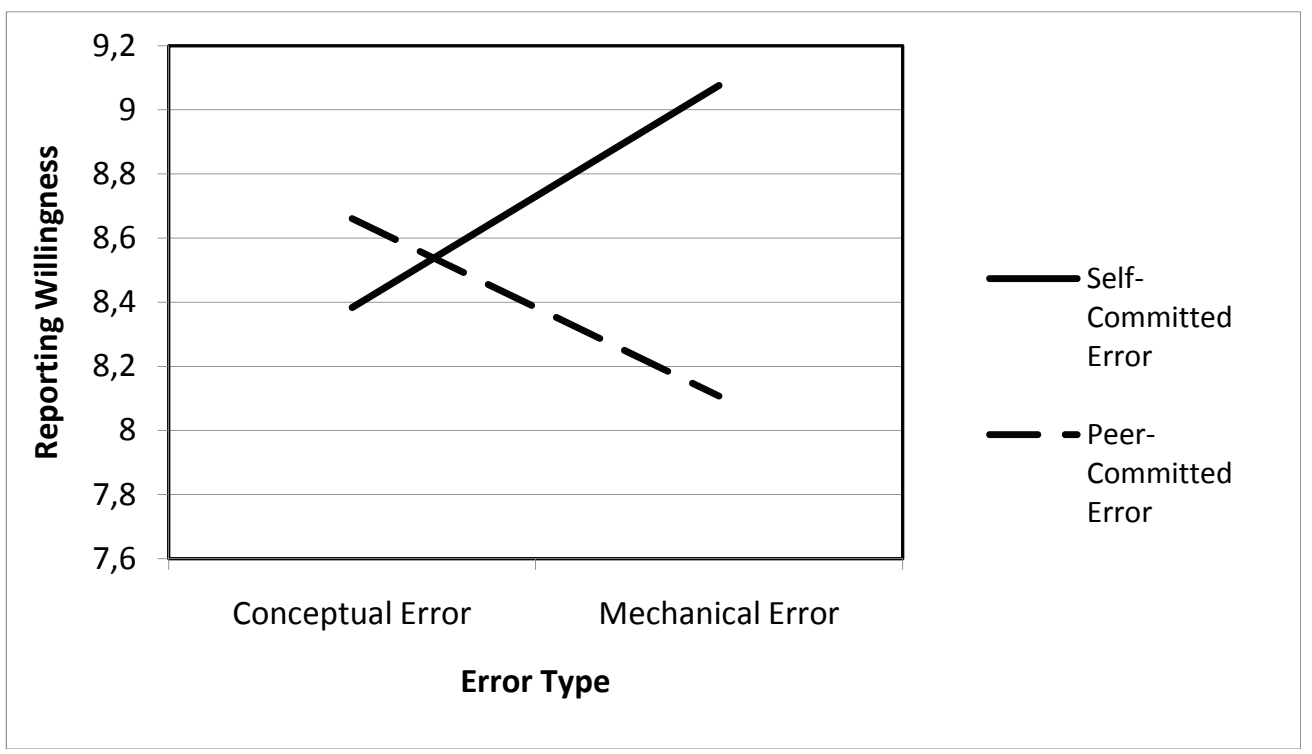




\section{Figure 3}

Panel A: Predicted Interaction Effect of Error-Management Climate x Error Originator on Report Willingness (H5)

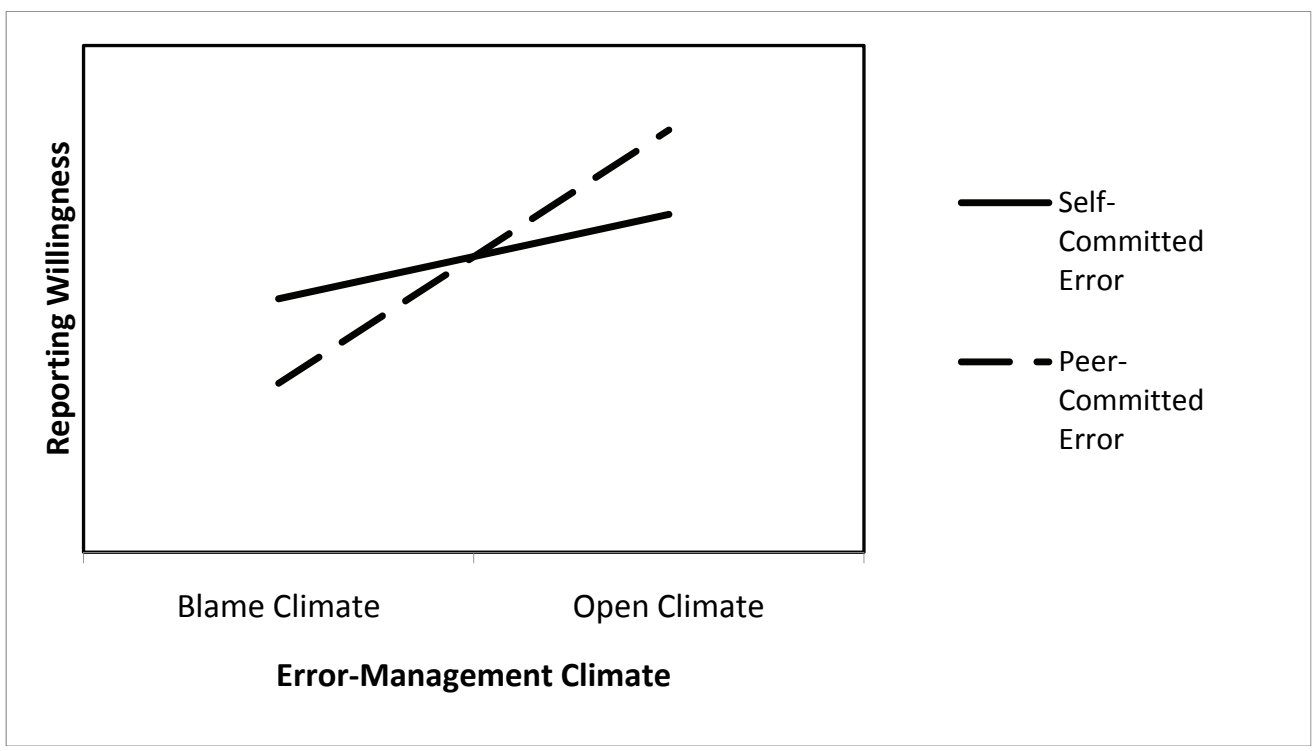

Panel B: Observed Interaction Plot: Error-Management Climate by Error Originator Report Willingness (Adjusted Means)

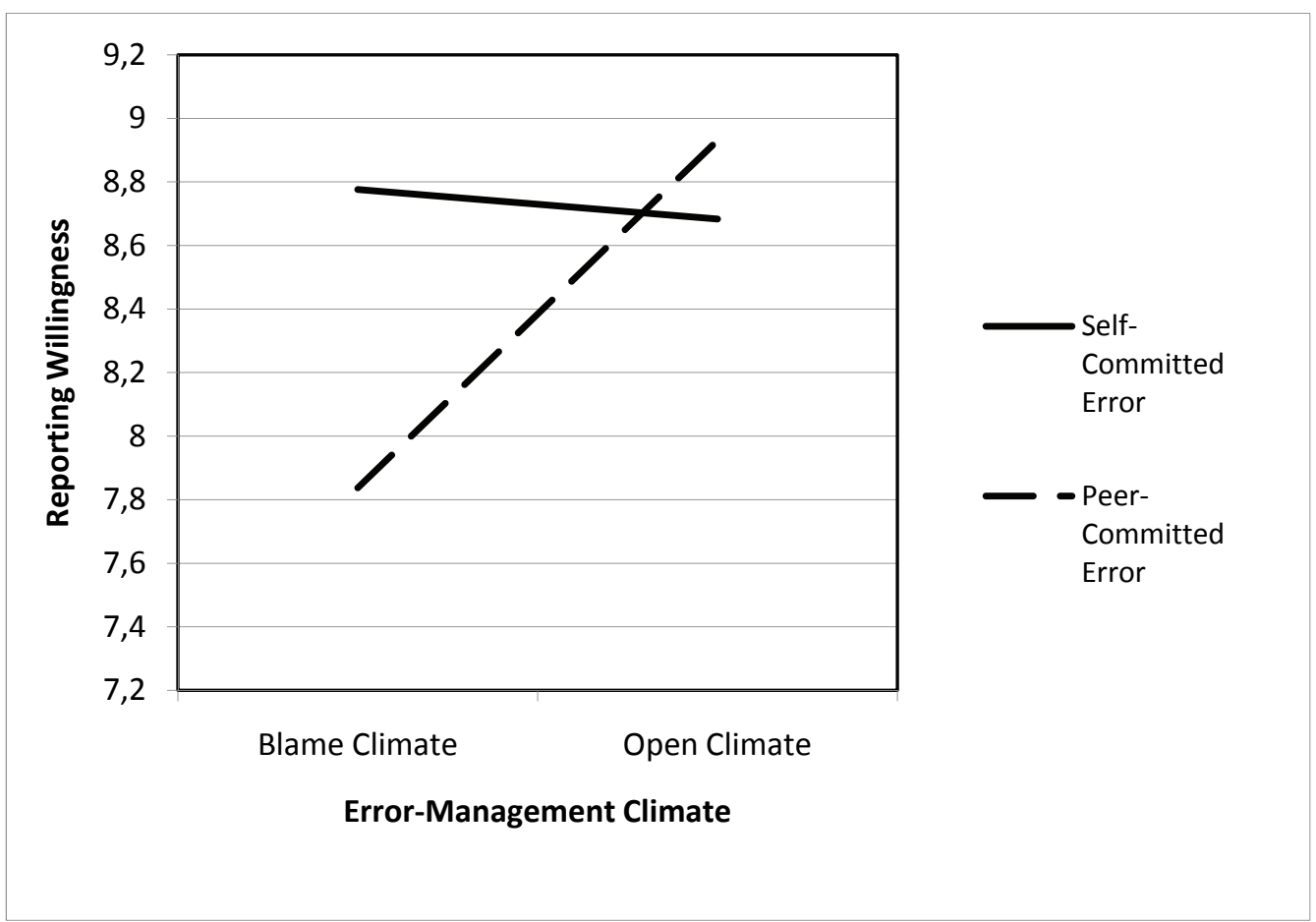

